\title{
SOBRE LA GEOMETRIA DE LOS CUENCOS LACUSTRES GLACIARES PIRENAICOS
}

M.A.Gil Saurí

Escuela Técnica Superior de Ingenieros de Caminos.Campus de Vera.46071-Valencia.

Palabras clave: Cuaternario,glaciarismo, morfogénesis, cuenco,geometría.

\section{ABSTRACT}

The Quaternary glacial morphogenetic system has produced overexcavated areas which, by the melting of the glaciar, give rise to lakes that, downstream, remain clo sed in by strong rocky thresholds or by moraines deposited by the glaciar.A mixed situation is also possible.

The morphogenetic analysis of different lakes in the Spanish Pyrenees has permitted a geometric characterization of these glacial cavities to be established. In this way, by means of the elaboration of hypsographic curves, the moșt con cordant mean form for these lake basins has been deduced.

\section{INTRODUCCION}

En España hay glaciares actuales en núcleos muy reducidos.Se acantonan en el Pirineo alrededor de $1053000 \mathrm{~m}$. de :altitud.Vestigios glaciares cuaternarios, fue

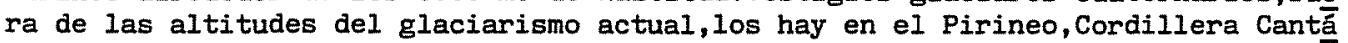
brica, Macizo Galaico,Cordillera Ibérica y Central y, en el Sur,Sierra Nevada.

El efecto principal de la morfogénesis glaciar fue la sobreexcavación. En muchas zonas sobreexcavadas, al desaparecer el glaciar, se produjeron lagos;éstos tie nen su origen en los valles, por donde discurren las lenguas glaciares, en los circos, donde se forman cuando el retroceso del hielo ha sido total.

El análisis morfométrico de distintas concavidades ha determinado la caracte rización geométrica de estas formas glaciares.Se ha particularizado en los lagos glaciares(Pirineos), especialmente en 67 de ellos se ha podido obtener toda suerte de datos.El estudio sobre 81 criptodepresiones individuales, pertenecientes a las antedichas masas acuíferas, se ha comparado con cifras y resultados de morfolo gía extranjera.

Por último, la formulación, que ha permitido una definición geométrica aproximada del cuenco lacustre medio glaciar pirenaico,facilita explicaciones sobre los distintos factores que intervienen en la morfogénesis glaciar.Asimismo, señalamos unas interesantes investigaciones a realizar,relativas a la comprobación obtenida en aiferentes ámbitos de actuación del glaciarismo cuaternario en nuestra Penínsu la.

Otro camino a seguir es el estudio de lagos y lagunas con distinto origen ge nético al glaciar; de este modo,será posible establecer una clasificación conjunta.

\section{AREA DE ESTUDIO}

Para el análisis morfológico se consideró conveniente limitar el campo de ac ción a la vertiente meridional de la Cordillera Pirenaica.Fue así,por un lado, porque la erosión glaciar adquirió gran extensión;por otra parte,se pudo disponer de mayor número de observaciones batimétricas, lo que facilitó el trabajo de campo.

El acervo principal empleado para nuestra investigación se circunscribió a ragón y Cataluña(provincias de Lérida y Huesca), fig. 1.

Los datos característicos de los ibones-apelativo con el que se conocen los lagos y lagunas de origen glaciar en Aragón(etimológicamente del francés medieval "iba" y del celta "avon", contracción de ambos vocablos, cuyo significado, respectivamente, es "agua" y"corriente de agua") - de la muestra:profundidad máxima H,super ficie $S_{H} y$ volumen $v_{\text {, }}$, todo referido al nivel del umbral natural del lago,se inclu yen en $\mathrm{H}_{\text {tabla de }} \mathrm{Ia}^{\mathrm{R}}$ página siguiente. 

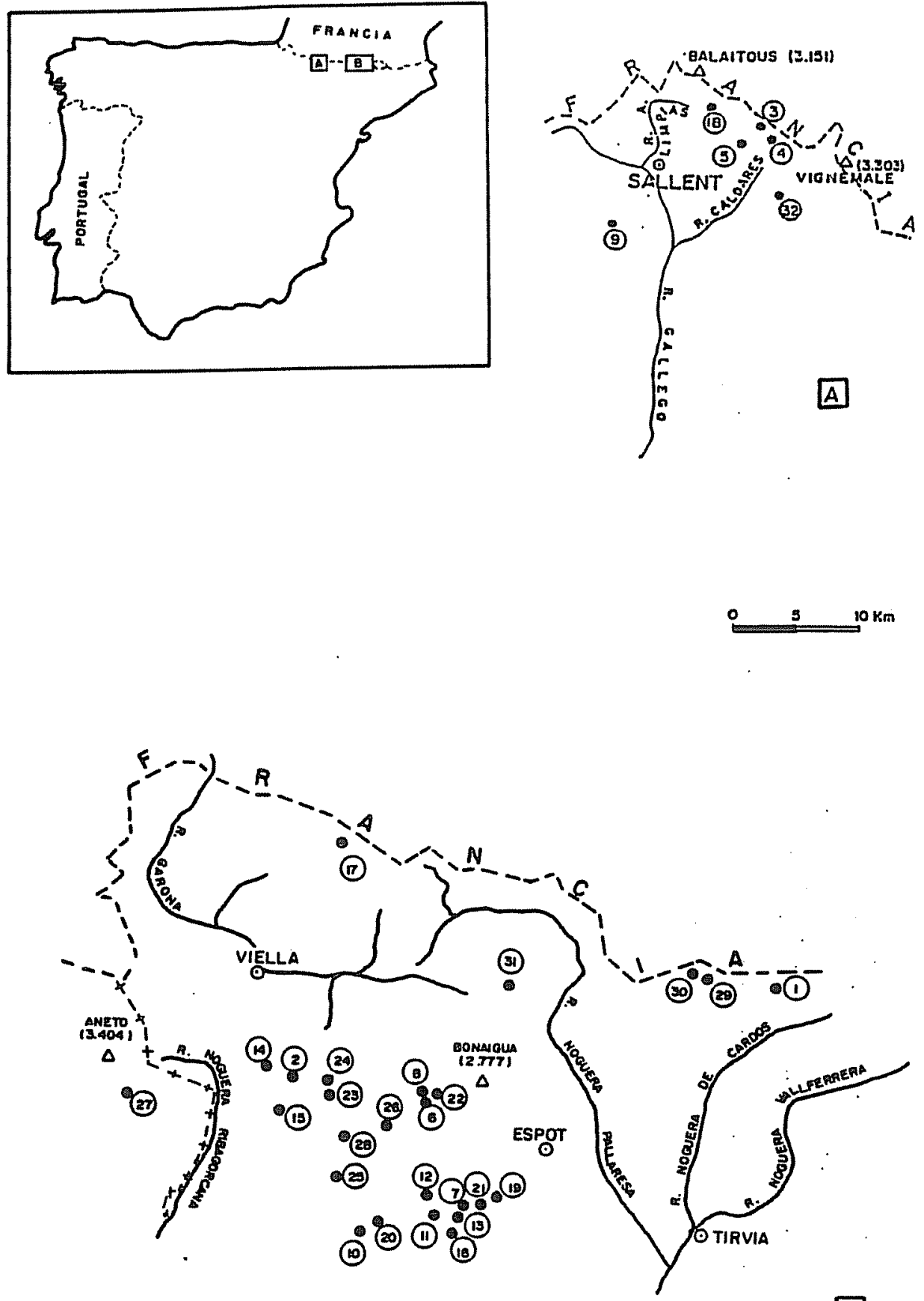

回

Fig.1.Distribución geográfica de los lagos glaciares de la muestra. 


\begin{tabular}{|c|c|c|c|c|c|}
\hline No(*) & NOMBRE DEL LAGO & ALTITUD (m.) & $H\left(m_{*}\right)$ & $\mathrm{S}_{H}\left(10^{4} \mathrm{~m}^{2}\right)$ & $V_{R}\left(10^{3} m^{3}\right)$ \\
\hline 1 & Certescans ( 3 ) & 2232.56 & 104.56 & 59.30 & 29449.612 \\
\hline 2 & Mar-Arties (3) & 2223.30 & 83.30 & 46.03 & 14219.430 \\
\hline 3 & Bramatuero-Inf. & 2311.00 & 18.00 & 10.75 & 684.345 \\
\hline 4 & Bramatuero-Sup. & 2526.00 & 30.00 & 23.60 & 3815.210 \\
\hline 5 & Azul-Inferior & 2375.00 & 25.00 & 4.08 & 589.738 \\
\hline 6 & Saburedo Mayor (2) & 2318.95 & 20.95 & 14.80 & 1268.875 \\
\hline 7 & Mar de Capdella & 2427.00 & 72.00 & 29.12 & 10784.276 \\
\hline 8 & Mayor de Colomers & 2082.15 & 24.15 & 8.27 & 843.381 \\
\hline 9 & Tramacastilla & 1680.70 & 4.70 & 2.32 & 35.335 \\
\hline 10 & Fosser (2) & 2122.95 & 34.95 & 11.15 & 2276.759 \\
\hline 11 & Colomina & 2411.20 & 64.20 & 12.32 & 3776.788 \\
\hline 12 & Mariolo & 2291.00 & 21.00 & 12.50 & 875.003 \\
\hline 13 & Frescau & 2418.30 & 33.30 & 6.82 & 1000.096 \\
\hline 14 & Tort de Rius & 2325.75 & 41.75 & 85.44 & 9206.437 \\
\hline 15 & Gémenes Inferior & 2233.00 & 19.00 & 8.14 & 751.087 \\
\hline 16 & Gémenes Superior & 2269.00 & 31.00 & 7.67 & 1232.207 \\
\hline 17 & Estany Gento(2) & 2132.00 & 22.00 & 21.81 & 2381.511 \\
\hline 18 & Liat & 2115.00 & 39.00 & 26.70 & 3805.081 \\
\hline 19 & Pecico Grande & 2332.00 & 38.00 & 9.14 & 1424.274 \\
\hline 20 & Negro de Peguera & 2333.00 & 69.00 & 28.70 & 10294.171 \\
\hline 21 & Tort de Capdella & 2316.00 & 15.00 & 5.15 & 327.877 \\
\hline 22 & Saburó & 2530.00 & 84.00 & 23.28 & 10106.543 \\
\hline 23 & Gerbé & 2178.00 & 50.00 & 15.36 & 3957.092 \\
\hline 24 & Travesany & 2244.40 & 26.40 & 11.09 & 1037.470 \\
\hline 25 & Tucmeneges Inf. & 2281.40 & 13.40 & 3.09 & 170.787 \\
\hline 26 & Cavallers & 1723.50 & 13.50 & 10.80 & 560.300 \\
\hline 27 & llong & 1980.00 & 12.00 & 8.10 & 489.281 \\
\hline 28 & Llauset & 2131.00 & 11.00 & 5.34 & 232.372 \\
\hline 29 & Negro de Bohí & 2147.00 & 27.00 & 9.74 & 840.649 \\
\hline 30 & Meriola & 2272.40 & 38.40 & 17.93 & 3026.486 \\
\hline 31 & Mayor de Sens & 2490.00 & 50.00 & 11.90 & 2847.579 \\
\hline \multirow[t]{24}{*}{32} & Airoto & 2202.80 & 40.80 & 19.40 & 4038.626 \\
\hline & Brazato Inferior & 2286.00 & 19.00 & 4.40 & 354.210 \\
\hline & Moncasau & 2022.00 & 4.00 & 2.24 & 58.608 \\
\hline & Ratera Grande & 2362.50 & 19.50 & 4.73 & 411.698 \\
\hline & Ratera Mediano & 2379.60 & 14.60 & 2.60 . & 156.319 \\
\hline & Ratera Pequeño & 2370.00 & 5.00 & 1.00 & 20.500 \\
\hline & Saburedo Superior & 2622.35 & 24.35 & 8.34 & 1064.991 \\
\hline & Closell & 2075.00 & 5.00 & 1.35 & 26.495 \\
\hline & Rumedo Superior & 2125.40 & 11.40 & 5.58 & 232.254 \\
\hline & Rumedo Medio & 2108.20 & 36.20 & 11.00 & 1642.930 \\
\hline & Llarg (3) & 2145.25 & 16.25 & 11.55 & 1108.219 \\
\hline & Ubago(2) & 2207.10 & 27.10 & 13.78 & 1466.073 \\
\hline & Cuvieso(2) & 2338.00 & 36.00 & 17.45 & 3810.620 \\
\hline & La Gola(2) & 2226.00 & 26.00 & 10.52 & 1296.167 \\
\hline & Arbé & 2110.80 & 42.80 & 5.42 & 1189.894 \\
\hline & Cabana de Liat & 2126.00 & 10.00 & 2.14 & 87.967 \\
\hline & Cap de Port & 2524.50 & 24.50 & 7.47 & 813.946 \\
\hline & La Llastra & 2429.00 & 15.00 & 2.29 & 171.065 \\
\hline & La Coveta & 2399.00 & 7.00 & 2.25 & 80.682 \\
\hline & La Cabana & 2379.00 & 9.00 & 2.28 & 92.309 \\
\hline & $\begin{array}{l}\text { Trullo } \\
\text { Ribereta Superior }\end{array}$ & $\begin{array}{l}2313.00 \\
2301.00\end{array}$ & $\begin{array}{l}17.00 \\
23.00\end{array}$ & $\begin{array}{l}2.37 \\
1.67\end{array}$ & $\begin{array}{l}195.920 \\
169.458\end{array}$ \\
\hline & Cabanes & 2192.00 & 54.00 & 6.81 & 1397.929 \\
\hline & Ribereta Inferior & 2247.20 & 53.20 & 7.76 & 1395.889 \\
\hline & $\operatorname{clot}(2)$ & 2120.45 & 12.45 & 3.16 & 115.120 \\
\hline
\end{tabular}




\begin{tabular}{|c|c|c|c|c|c|}
\hline №.(*) & NOMBRE DEL LAGO & $\operatorname{ALTITUD}(\mathrm{m})$. & $\mathrm{H}(\mathrm{m})$. & $\mathrm{S}_{\mathrm{H}}\left(10^{4} \mathrm{~m}^{2}\right)$ & $v_{R}\left(10^{3} m^{3}\right)$ \\
\hline & Naorte & 2150.00 & 12.00 & 4.19 & 291.032 \\
\hline & Manyera & 2164.75 & 4.75 & 2.13 & 75.900 \\
\hline & Rumedo Inferior & 1999.20 & 19.20 & 7.17 & 706.082 \\
\hline & Tucmeneges Sup. & 2317.25 & 39.25 & 13.29 & 1597.585 \\
\hline & Baborte & 2333.20 & 31.20 & 6.78 & 1033.297 \\
\hline & Pulgar & 2424.00 & 20.00 & 4.83 & 434.848 \\
\hline & Gallina & 2269.00 & 16.00 & 4.49 & 377.742 \\
\hline & Caldas (2) & 2184.00 & 16.00 & 3.37 & 191.114 \\
\hline & Rius-A & 2316.00 & 8.00 & 0.75 & 31.487 \\
\hline & Rius-B & 2316.00 & 20.00 & 4.30 & 397.877 \\
\hline & Malniu & 2250.00 & 15.00 & 8.24 & 502.461 \\
\hline
\end{tabular}

(*) Corresponde a la numeración del plano de situación de la Fig.1

(n) Señala el número de depresiones sumergidas en cada lago.

\section{MATERIAL Y METODOS}

Se ha partido para cada lago de la muestra de una cartografía detallada.Tras la elaboración de las batimetrías correspondientes $y$, teniendo en cuenta los antecedentes históricos, se ha escogido la metodología, derivada de las curvas características de los lagos, como la mas adecuada para identificar geométricamente la cu beta lacustre.

Desde el primer tercio de la centuria actual, numerosos estudios se han reali zado sobre morfometría en ríos-caracterización de la morfologia interna en embalses- y en lagos-significación de los parámetros definidores del cuenco lacustre-. La clasificación geométrica de embalses, dentro de la morfogénesis fluvial, la analizó Garret,Hillman y Sutherland(1932). Análogos planteamientos matemáticos fue ron empleados por Sáenz García(1934)en España.

Por otro lado, las observaciones sobre morfología de lagos no quedaron sistematizadas hasta G.E.Hutchinson(1957). En España, el estudio morfológico de Nussbaum (1934) sobre los lagos del Pirineo es intuitivo pero poco riguroso.Pardo(1948), fa cilita datos sobre la morfometría de mas de 2400 lagos españoles sin proporcionar detalles de la morfología interna.

En este mismo contexto, Neumann(1959) señala un hito importante en las averiguaciones que hasta el momento habian tenido lugar para dar con la forma media de la concavidad lacustre. Sugiere una sinusoide eliptica como la geometría que, por término medio, mas se ajusta al cuenco lacustre-lagos de cualquier tipo-.La antedi cha geometría no es casaal : relación profundidad media( $\bar{D}) /$ profundidad máxima(D $)$ para este cuerpo es de 0.464.Por otra parte, este mismo autor trabajando con datos relativos a 107 lagos (90\% de origen glaciar) repartidos por el.mundo entero-lista do facilitado por G.E.Hutchinson(1903-1991), pudo elegir el valor 0.467 , para estos lagos, como promedio.

La relación aproximadamente lineal entre la profundidad media y la profundidad máxima con valor 1/2 (Neumann,1959), dio lugar a la elaboración de análoga representación para las 81 concavidades de la muestra pirenaica,fig.2.Advertida la similitud entre gráficos y razones-hay que considerar que un gran porcentaje estuي diado por Neumann tiene origen glaciar- se pensó en establecer una metodología mu cho mas exhaustiva que pudiera caracterizar geométricamente el cuenco lacustre pi renaico.En este sentido, se recurrió a las curvas características.Es decir, curvas que,para cada altura de agua o profundidad, relacionan la superficie horizontal y el volumen en función de la misma. La primera curva recibe tambien la denominación de hipsográfica(del griego hypsos, altura). Determinadas, para cada una de las 81 cu betas de la muestra, las precitadas líneas, se ensayaron familias de curvas teóricas con la finalidad de buscar el mejor ajuste a las mencionadas curvas reales. 


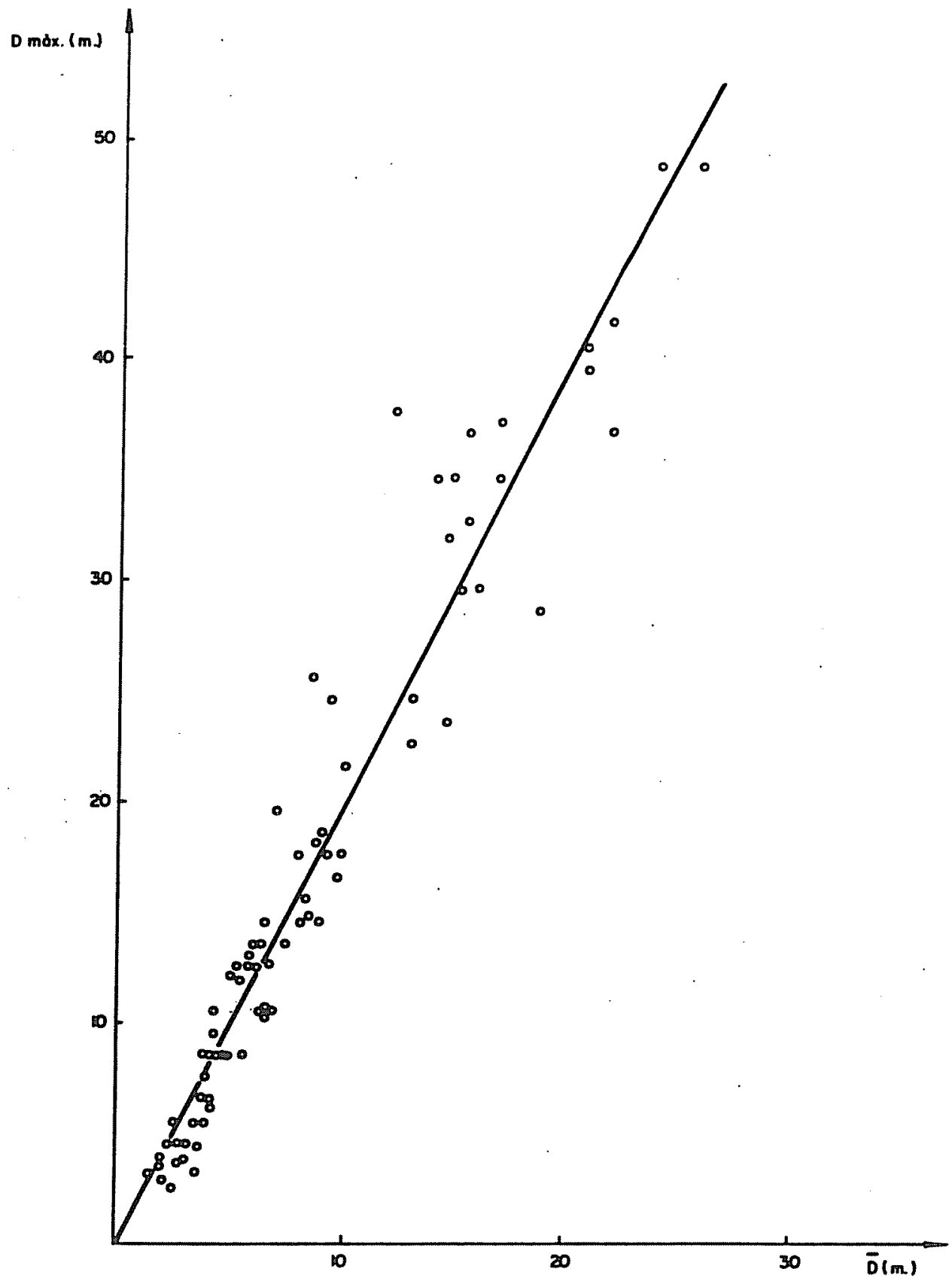

Fig.2. Correlación entre la profundidad media $\bar{D}$ y la profundidad máxima $D_{\text {máx }}$ para el cuenco glaciar pirenaico. 


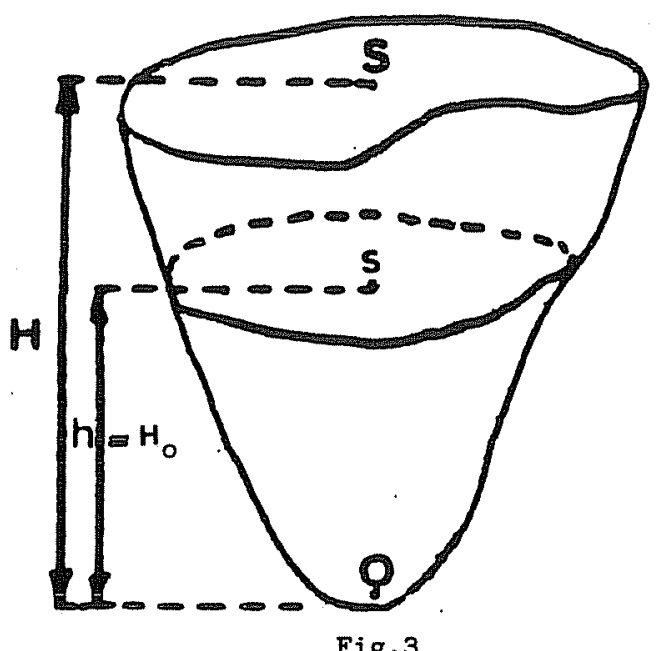

\section{RESULTADOS}

La curva teórica representativa del comportamiento real resultó ser una fami lia de parábolas de grado(n).Los distintos exponentes son números reales. Dada la gran cantidad de datos de la muestra, la determinación de la bondad del ajuste requirió tratamiento informático.

Ahora bien,en una primera aproximación,Sutherland(1932) que resulta ser sufi ciente, la curva tipo admite la simplificación siguiente:s=kh ${ }^{\mathrm{h}}$. El exponente es el mayor de los correspondientes a la familia de parábolas anterior.

Le curva característica de volumen se obtiene por integración de la anterior: $v=\mathrm{kh}^{\mathrm{n}+1} /(\mathrm{n}+1)$. En consecuencia, para la profundidad máxima $H$-valores $S_{H}$ y $v_{R}$-ver ta bla-se tiene: $V_{P}=S_{H}(H /(n+1))$.

El valor medio de la muestra $\bar{m}=n+1$ result máxima $H$, se cumple $: \bar{D} / \mathrm{Dm}=1 / \overline{\mathrm{m}}=0.4632$, es notorio que la cifra obtenida es cercana $a$ la de Neumann, lo que confirma-para cualquier altura de agua-la aproximación de la concavidad glaciar pirenaica-forma media-al paraboloide elíptico.

Con los cálculos efectuados, el cuenco lacustre glaciar pirenaico responde a una forma media cuyas características geometricas son:

$\mathrm{g}=\mathrm{k} \mathrm{h}^{1.1591}$

$v=k \cdot h^{2.1591}$

$\mathrm{H}_{0}=0.6835 \mathrm{H}$

El parámetro H -fig.3-representa la altura a la que está el centro de gravedad del cuerpo geŏmétrico sobre el punto mas bajo. En función de la profundidad máxima $H_{0}$ el valor de $H_{0}=H(n+1) /(n+2)$.

\section{DISCUSION}

E1 planteamiento matemático efectuado-adecuar convenientemente una ley parabólica a las curvas características reales-presenta errores aceptables, tanto por lo que se refiere a cada curva hipsogrffica de la muestra(2\%), como por lo que res pecta al valor medio(15\%, evidentemente mayor).

Los valores de $\bar{m}=2.1591$ (intervalo de oscilación $2 ; 2.3$ ), permiten apuntar una gran incidencia del fenómeno concreto de la sobreexcavación glaciar frente a factores que intervienen en el proceso general.Sin embargo, es necesario analizar la influencia de éstos en el campo de variacion de-m-. 
A este respecto, Nussbaum(1934)sostiene que los lagos mas profundos se encuen tran en zonas de pizarras, ya que son mas fácilmente erosionables.

De los resultados obtenidos se puede colegir que la formación de las concavi dades depende del tamaño del glaciar-coeficientes $\mathrm{k}$ y k'-.Por consiguiente, cabe ù na relación homotética entre curvas hipsográficas-para la misma $\underline{h}$ e idéntico m.Además, la influencia de la litología en la morfología fluvial ya se ha comprobàdo, Gómez Navarro(1966).

otro elemento de incidencia en la modificación de $\bar{m}$ es la tectónica. Referente a ello,el geomorfólogo centroeuropeo afirma:"Uno de los problemas mas interesantes de la limnología glaciar es el de la formación de lagos.El sistema erosivo viene alterado por el ángulo del plano inclinado por donde discurre el glaciar, no obstante, los grandes lagos alpinos no pueden explicarse satisfactoriamente sin re currir a dislocaciones tectónicas.Por eso se ha intentado atribuir el mismo origen a los lagos pirenaicos;sin embargo,el único lago que puede formarse así es el de Bramatuero(Alto Caldares,fig.1)".

Esta contribución estructural en la morfogénesis glaciar, no solo es mantenida sino reforzada (E. Martínez de Pisón, M. Arenillas Parra,1977): En el Pirineo, los lagos de circo son, como norma, claramente mas redondeados que los de fondo de va$11 \mathrm{e}$, lo cual tiene que tener, fundamentalmente, una explicación tectónica;los circos, en los que se produce el desalojo de los materiales pétreos por el glaciar, suelen quedar definidos por la intersección de distintas fallas o fracturas, mientras que en el fondo del valle la estructura dominante resulta ser casi siempre la que define el propio valle.

Otro elemento modificativo de $\bar{m}$ es el retoque postglaciar que, obviamente,pro pició que las paleoformas, originadas por el hecho diluvial, no tuvieran estos par自 metros sino unas ligeras alteraciones de los mismos(Gil Saurí,1987).

\section{CONCLUSTONES}

El cuenco lacustre glaciar pirenaico puede identificarse con una forma geomé trica en la que la superficie horizontal y volumen en función de la profundidad $\overline{0}$ calado tiene las expresiones matemáticas sigulentes:

$$
s=k h^{1.16} v=k^{\prime} h^{2.16}
$$

La geometría de las criptodepresiones lacustres pirenaicas de origen glaciar es similar al paraboloide elíptico, exponente $\bar{m}=2$.

A efectos de cubicación del lago-determinación de su volumen-es posible apli car la fórmula $v_{B}=S_{H}(H / \bar{m})$ con los valores obtenidos, cálculo de $S_{H}$ en el plano y dé finición de $\mathrm{H}$, ya sea, mediante unas cuantas medidas batimétricas, o bien, por extra polación, a partir de la topografía externa mediante cortes transversales.

\section{AGRADECIMIENTOS}

Este trabajo surgió estando el autor en la Cátedra de Geología Aplicada a la Ingeniería de la Escuela Técnica Superior de Ingenieros de Caminos,Canales y Puer tos de la Universidad Politécnica de Madrid donde,merced a una beca y la ayuda del Profesor D.Clemente Sáenz Ridruejo,se pudo iniciar una investigación sobre la morfología de los lagos y lagunas glaciares españolas.

Es por ello que el autor desea expresar, en especial, su gratitud al mencionado Profesor y sus colaboradores, cuya orientación, sugerencias y consejo en cada mo mento han sido fundamentales para iniciar estas investigaciones que esperamos coñ cluyan con una clasificación genética de las diferentes formas lacustres.

No sería justo terminar este epígrafe sin manifestar el recorocimiento de la labor desarrollada por las personas que, durante nuestra estancia por las diversas zonas estudiadas, han utilizado parte de su tiempo $y$ nos lo han dedicado. 


\section{BIBLIOGRAFIA}

Burgis,M.J.;P.Morris.1987. The Natural History of Lakes.Cambridge University.218. Dussart, B.1966.Limnologie.Gauthier-Villars.677.

Gil Sauri,M.A.1987.Morfometría de los lagos glaciares pirenaicos.Eria.83-86. Gómez Navarro,J.L.1966.Saltos de Agua y Presas de Embalse.Dossat.T2.1522-1535. Hakanson, L.1981. A manual of lake morphometry.Springer-Verlag.77.

Hutchinson, G.E.1975. A treatise on limnology. Jhon-Wiley.v1.653.

Margalef, R.1975. Introducción al estudio de los lagos pirenaicos. Naturalia Hispánica. (4). 45.

Martínez de Pisón,E.;M.Arenillas.1977.E1 problema de la sobreexcavación glaciar. Estudios Geográficos. (145).407-434.

Neumann,J.1959.Maximum depth and average depth of lakes.Journal Fish.Res.Bd.16(6) 923-927.

Nussbaum,F.1934.Die seen der pyrenäen.Mitt.Naturforsch.Gesells.184.

Pardo,L.1948. Cat́́logo de lagos españoles. In.Forest.Invest.Experienc. (41).522.

Saénz García,C.1934.Forma empírica de las curvas características de los embalses. Actas XIV Congreso de la Asociación Española Progreso Ciencias.9.

Solé Sabarís,L.1936.Els llacs dels Pirineus segons Nussbaum.Butlletí Institut Ca talá d'Historia Natural. (36) .107-115.

Sutherland, R.A.1932.Some aspects of water conservation.Transactions-A.S.C.E.157. 